\title{
The impact of socioeconomic status and
}

\section{multimorbidity on mortality: a population-based cohort study}

\author{
Nikoline Lund Jensen ${ }^{1,2}$ \\ Henrik Søndergaard \\ Pedersen' \\ Mogens Vestergaard', 2 \\ Stewart W Mercer $^{3}$ \\ Charlotte Glümer ${ }^{4}$ \\ Anders Prior ${ }^{1,2}$
}

'Research Unit for General Practice, ${ }^{2}$ Section for General Medical Practice, Department of Public Health, Aarhus University, Aarhus, Denmark; ${ }^{3}$ General Practice and Primary Care, Institute of Health and Wellbeing, University of Glasgow, Glasgow, Scotland; ${ }^{4}$ Research Centre for Prevention and Health, The Capital Region of Denmark, Glostrup, Denmark

Correspondence: Nikoline Lund Jensen Research Unit for General Practice and Section for General Medical Practice, Department of Public Health, Aarhus University, Bartholins Allé 2, 8000

Aarhus, Denmark

Tel +45 51898018

Email nikolinelundjensen@ph.au.dk
This article was published in the following Dove Press journal:

Clinical Epidemiology

10 May 2017

Number of times this article has been viewed

Objective: Multimorbidity (MM) is more prevalent among people of lower socioeconomic status (SES), and both MM and SES are associated with higher mortality rates. However, little is known about the relationship between SES, MM, and mortality. This study investigates the association between educational level and mortality, and to what extent MM modifies this association.

Methods: We followed 239,547 individuals invited to participate in the Danish National Health Survey 2010 (mean follow-up time: 3.8 years). MM was assessed by using information on drug prescriptions and diagnoses for 39 long-term conditions. Data on educational level were provided by Statistics Denmark. Date of death was obtained from the Civil Registration System. Information on lifestyle factors and quality of life was collected from the survey. The main outcomes were overall and premature mortality (death before the age of 75).

Results: Of a total of 12,480 deaths, 6,607 (9.5\%) were of people with low educational level (LEL) and 1,272 (2.3\%) were of people with high educational level (HEL). The mortality rate was higher among people with LEL compared with HEL in groups of people with 0-1 disease (hazard ratio: $2.26,95 \%$ confidence interval: $2.00-2.55$ ) and $\geq 4$ diseases (hazard ratio: $1.14,95 \%$ confidence interval: 1.04-1.24), respectively (adjusted model). The absolute number of deaths was six times higher among people with LEL than those with HEL in those with $\geq 4$ diseases. The 1-year cumulative mortality proportions for overall death in those with $\geq 4$ diseases was $5.59 \%$ for people with HEL versus $7.27 \%$ for people with LEL, and 1-year cumulative mortality proportions for premature death was $2.93 \%$ for people with HEL versus $4.04 \%$ for people with LEL. Adjusting for potential mediating factors such as lifestyle and quality of life eliminated the statistical association between educational level and mortality in people with MM.

Conclusion: Our study suggests that LEL is associated with higher overall and premature mortality and that the association is affected by MM, lifestyle factors, and quality of life.

Keywords: multimorbidity, socioeconomic status, social epidemiology, inequality in health, mortality, population-based cohort study

\section{Introduction}

The pattern of health challenges has changed over the recent decades. Chronic diseases now account for majority of deaths in developed countries. ${ }^{1}$ The rising prevalence of multiple chronic diseases in an increasingly aging population ${ }^{2,3}$ poses considerable challenges in the highly specialized health care systems, which primarily focus on treating single diseases. ${ }^{4-6} \mathrm{MM}$ is associated with several adverse health outcomes, including high health care utilization, ${ }^{7}$ unplanned hospital admission, ${ }^{8}$ lower quality of life, ${ }^{9-13}$ mental health disorders, ${ }^{14}$ lower functional level, ${ }^{15-17}$ higher prevalence of pain, ${ }^{18}$ and higher mortality rates. ${ }^{19-21}$ 
Several cross-sectional studies have reported that the prevalence of MM is socially patterned. This has been shown by using various deprivation indices as proxies for socioeconomic status (SES) ${ }^{22-24}$ Barnett et al found that MM occurs 10-15 years earlier in people living in deprived areas of Scotland compared with affluent areas. Socioeconomic deprivation is particularly associated with $\mathrm{MM}$ involving mental health disorders. ${ }^{14}$ Other studies have investigated educational level as a risk factor for developing long-term disease, and these have confirmed that MM is more common among people with low SES. ${ }^{25-28}$

It is well established in many European countries that people with low educational level (LEL) (as a measure for SES) have higher mortality than those with high educational level (HEL). This difference illustrates the considerable inequalities in health. ${ }^{29-32}$ As knowledge on the association between educational level, MM, and mortality is limited, prospective studies are warranted on the impact of SES on the long-term survival in people with MM. ${ }^{33}$

The objective of this study was to examine the impact of educational level on all-cause mortality when taking MM status into account. Poor lifestyle choices are known to be associated with MM, ${ }^{34}$ low SES, ${ }^{35}$ and high mortality. ${ }^{36}$ This underlines the importance of including relevant lifestyle factors when answering the research question. We used a large population-based cohort comprising a representative sample of the Danish population to compare information on socioeconomic factors, morbidity burden, and specific lifestyle factors. A 4-year follow-up study was conducted, and additionally, lifestyle was further investigated for a subgroup. We hypothesized that low SES, chronic disease burden, and poor lifestyle choices would interact and contribute to an impaired prognosis in people with MM.

\section{Methods}

\section{Study design}

This nationwide population-based cohort study with prospectively recorded data was conducted using information on people who were invited to participate in the Danish National Health Survey $2010 .^{37}$ The survey population consisted of a random nationally representative sample of all Danish citizens from each of the five Danish regions and an additional national sample invited by the National Institute of Public Health. Information collected through a paper- and web-based survey questionnaire was linked to nationwide health registries at the personal level by using the unique 10-digit civil personal registration (CPR) number assigned to all Danish residents upon birth or immigration. ${ }^{38}$
Register data were collected prospectively. All participants entered the study on May 1, 2010 (baseline), at which time all questionnaires were collected. They were followed until death or emigration, or at the endpoint of the study (March 2014, 29), whichever came first.

\section{Study population}

We included all people who were invited to participate in the survey. Inclusion criteria were 25-89 years of age at baseline and residing in Denmark since January 1, 1995, to ensure the most complete register information. Participants with missing information on educational level $(n=2,956)$ were excluded from the analyses. The study population $(n=239,547)$ was subsequently divided into two sub-cohorts: survey respondents and nonrespondents, (Figure 1) leaving us with three cohorts; two sub-cohorts contained within the study population cohort.

\section{Exposure \\ MM status}

The study population was categorized with MM if they had two or more of the selected 39 long-term conditions, which have previously been defined and used in Danish cohorts ${ }^{39}$ based on recommendations in systematic reviews. ${ }^{40,41}$ The conditions were identified by combining data from the Danish National Patient Register, ${ }^{42}$ the Danish Cancer Register, ${ }^{43}$ the Danish Diabetes Register, ${ }^{44}$ the Danish Psychiatric Central Register, ${ }^{45}$ and the Danish National Prescription Registry ${ }^{46}$ from 1995 up until the baseline of study. Conditions were considered to be either physical or mental..$^{39}$ We divided the study population into three MM groups, which included both physical and mental conditions, at baseline: no (zero to one condition), moderate (two to three conditions), and severe MM (four or more conditions).

\section{Educational level}

Data on educational level were collected from Statistics Denmark $^{47,48}$ at the baseline of the study. Educational level was categorized according to United Nations Educational, Scientific and Cultural Organization's International Standard Classification of Education. ${ }^{49}$ Based on information on the highest degree attained, the study population was divided into three groups according to years of schooling ${ }^{50}$ : 1) LEL: $\leq 10$ years (e.g., no formal education, elementary school), 2) medium educational level (MEL): $>10$ and $\leq 15$ years (e.g., high school), and 3) HEL: $>15$ years (e.g., primary school teacher, journalist, physiotherapist, or physician). 


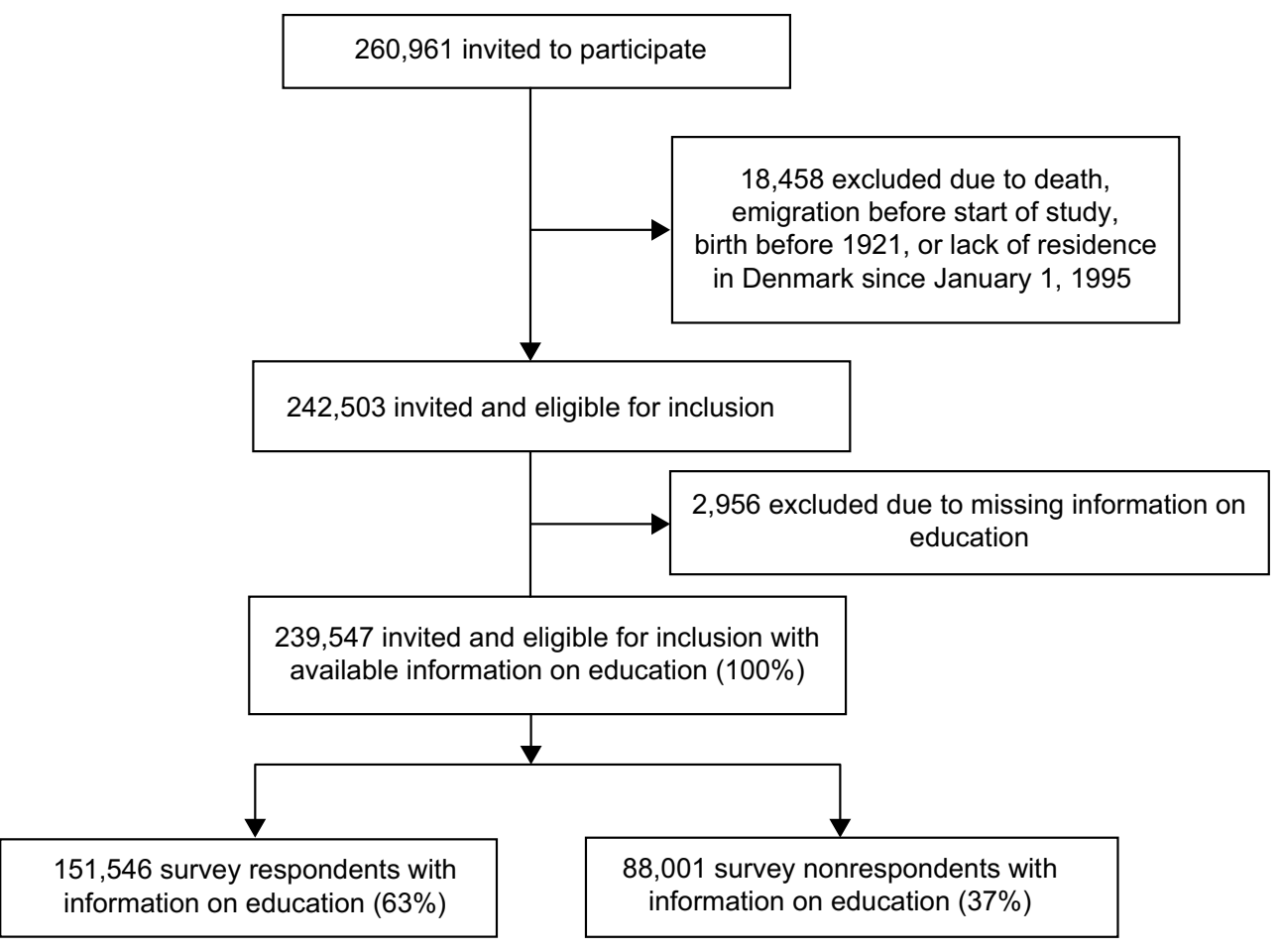

Figure I Flowchart of participants.

\section{Outcome}

\section{Information on death}

The primary outcome was all-cause mortality. Information on death was obtained from the Danish Civil Registration System,${ }^{38}$ which holds continuously updated information on vital status. Information on death is updated on a daily basis. In a sensitivity analysis, we investigated premature mortality defined as death before the age of 75 years.

\section{Covariates}

\section{Self-reported covariates}

Self-reported baseline characteristics were obtained on the sub-cohort of respondents from the Danish National Health Survey (Figure 1). We included information on lifestyle factors in groupings: physical activity (light or no physical activity, moderate physical activity of $\geq 4 \mathrm{~h} /$ week, and hard physical activity of $\geq 4 \mathrm{~h} /$ week $)$, alcohol habits $(<7 /<14$, 7-14/14-21, and >14/>21 units per week for females/males), smoking status (never, former, and current), body mass index (underweight, i.e., $<18 \mathrm{~kg}$; normal weight, i.e., $18-25 \mathrm{~kg}$; overweight, i.e., $25-30 \mathrm{~kg}$; or obese, i.e., $>30 \mathrm{~kg}$ ), ethnicity (Danish, other western background, or other), and self-rated physical and quality of life (measured by the Short-Form Health Survey (SF-12) $)^{51}$ by scores $0-100$, with 100 being the best possible state of health).

\section{Other covariates}

From the Danish Civil Registration System, ${ }^{38}$ we obtained individual information on gender, date of birth, and civil status (cohabiting or single). The data were fully anonymized.

The study did not need approval from the regional ethics committee, but it was approved by the Danish Data Protection Agency (file number 2013-41-1719).

\section{Statistical analysis}

The demographic characteristics of both study cohort and respondent cohort according to educational level were evaluated at baseline.

Hazard ratios (HRs) of all-cause mortality with 95\% confidence intervals (95\% CIs) were calculated using a Cox proportional hazards model with age as the timescale. We checked the proportional hazards assumption using log $(-\log [$ survival $])$ plots, and we saw no violations.

We developed four adjustment models. In the crude model (M1), we included the intrinsic correction for age used as timescale. In the second model (M2), we further adjusted for sex and cohabitation status. In the third model (M3), we additionally adjusted for all physical and mental conditions from the registers in the form of 39 dummy variables to account for the differences in influence on mortality caused by each condition. In a fourth model (M4), 
which was only applied to the respondent cohort, we further adjusted for self-reported lifestyle factors and quality of life (SF-12). In the last model, we imputed missing survey data on lifestyle and socioeconomic factors in a chained equations model. This is a method based on sequential multivariate regressions on missing parameters that allows us to impute multiple variables. We included all our analysis parameters, outcome variable, and time variable in the imputation model. Ordinal logistic regression was used for categorical variables and predictive mean matching for the SF-12 variables. We estimated that 20 imputation sets would be sufficient according to guidelines, and the resulting estimates were combined using Rubin's rules. Multiple imputation operates under the assumption that given the variables used in the imputation procedure, the missing data are missing at random, which means that the probability that a value is missing depends only on observed values and not on unobserved values. ${ }^{52,53}$

The association between educational level and mortality was examined by setting the highest educational level as reference and applying M1, M2, and M3. The association between MM and mortality was examined by setting " $0-1$ condition" as reference and applying M1 and M2. We then examined the association between educational level and mortality across MM groups by using M1, M2, and M3, and additionally applying M4 for survey respondents. Effect modification was assessed by testing for multiplicative interaction using a likelihood ratio test. The point estimates and the corresponding 95\% CIs were presented graphically; point estimates for educational level were adjusted using M3 and calculated for each MM group, and the ratios between the MM groups were adjusted using M2 and scaled by risktime-weighted average HRs within each MM group using the non-MM group as the reference group. This scaling was done to reflect the actual observed differences in condition status between MM groups (Figure 2).

Using a standard Kaplan-Meier approach, we calculated the cumulative mortality proportion (CIP) for death after 1year in all combinations of educational level across each MM group (CIP after 1 year $[\mathrm{CIP}]_{1 \mathrm{y}}$ ). To assess the magnitude of educational level across MM groups in absolute numbers, we multiplied the $\mathrm{CIP}_{1 \mathrm{y}}$ with the adjusted attributable fraction (HR-1)/HR and yielded the excess CIP ${ }_{1 y}{ }^{54}$

Multiple sensitivity analyses were performed. In one sensitivity analysis, we examined the effect of educational level on premature mortality across MM groups. In this analysis, individuals were censored at the time of their 75 th birthday; once a person turned 75 , he/she was no longer at

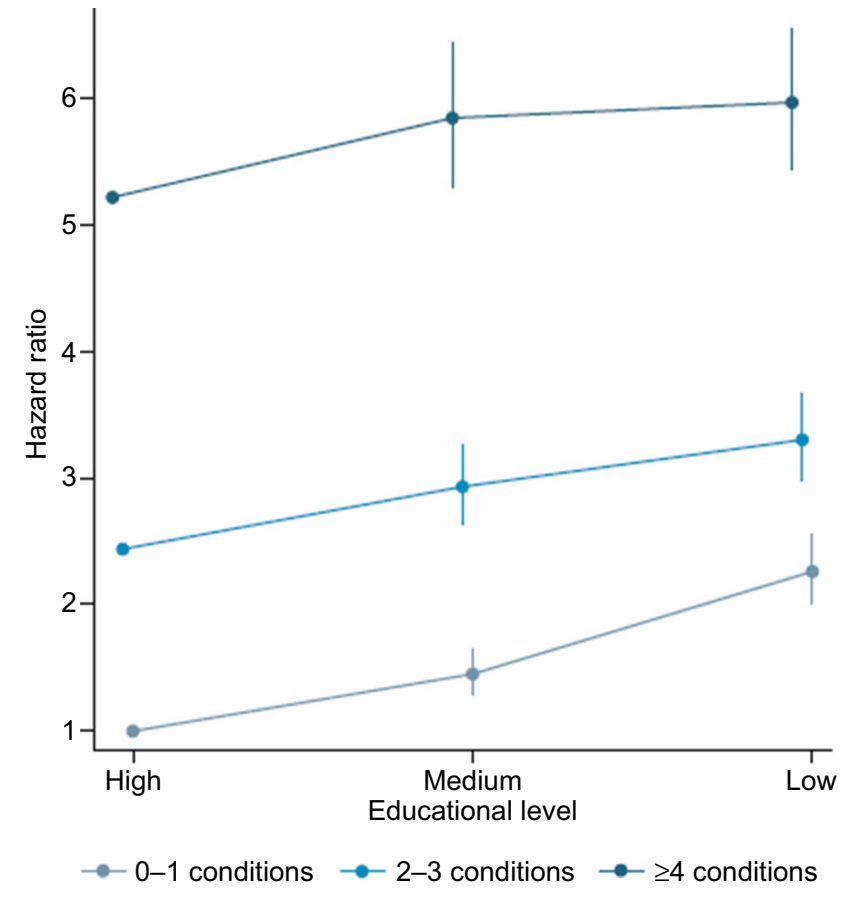

Figure 2 Association between educational level, multimorbidity group, and mortality hazard ratio.

risk of dying prematurely, that is, turning 75 years old is a competing risk of dying prematurely. As a consequence of this, the $\mathrm{CIP}_{1 \mathrm{y}}$ was estimated using a competing risk method in this sensitivity analysis. ${ }^{55,56}$ As in the main analysis, HRs were calculated using a standard Cox proportional hazards model with age as the timescale.

In another analysis, we excluded people $<35$ years at baseline to test the hypothesis on a cohort who more certainly had completed their education.

We also examined whether the effect of educational level across MM groups in the entire cohort differed by sex and age registered using stratified analyses. A likelihood ratio test was applied to test for multiplicative interaction across the different strata.

Respondent and nonrespondents were compared regarding demographic characteristics and mortality. The effect of educational level across MM groups was examined and compared between respondents and nonrespondents by using M3.

All analyses were performed using Stata 13 (Stata Corporation, College Station, TX, USA).

\section{Results}

We followed 239,547 individuals invited to participate in the Danish National Health Survey 2010 for a total of 910,400 person-years at risk (mean follow-up time: 3.8 years; Figure 1). In the study cohort, 29\% had LEL, $48 \%$ 
had MEL, and 23\% had HEL at baseline. LEL was more common among participants, who were elderly and single, had $\geq 4$ diseases, and had a psychiatric condition (Table 1).

The absolute number of long-term conditions increased with decreasing educational level (Figure 3).

\section{Association between educational level and mortality across MM groups}

We identified 12,480 deaths, of which 9,742 (78\%) were among people with MM. Among people with LEL 6,607 people (9.5\%) died, among people with MEL 4,601 (4.0\%) died, and among people with HEL 1,272 (2.3\%) died during follow-up.

The mortality increased with decreasing educational level and with increasing number of underlying diseases. The association between educational level and mortality measured on the relative scale (HRs) attenuated with increasing level of MM; the $P$-value for interaction was $<0.001$ (Table 2). The mortality rate was $126 \%$ higher among people with LEL compared with those with HEL in the group of people with 0-1 conditions (HR: 2.26, 95\% CI: 2.00-2.55), whereas the corresponding estimates were $14 \%$ higher among people with $\geq 4$ diseases (HR: 1.14, 95\% CI: 1.04-1.24) after adjusting for age, sex, cohabitation status, and the 39 conditions (M3). However, the absolute number of deaths was higher among people with LEL than those with HEL in all MM groups; for those with $0-1$ conditions, 335 persons $(0.8 \%)$ with HEL died versus 1,293 (3.4\%) persons with LEL. For those with $\geq 4$ diseases, 514 persons $(18.3 \%)$ with HEL died $\left(\mathrm{CIP}_{1 \mathrm{y}}\right.$ :
$5.59 \%)$ versus 3,245 persons $(25.9 \%)$ with LEL (CIP ${ }_{1 y}$ $7.27 \%$; Figure 4).

Theoretically, had those with LEL had the same risk of dying as those with HEL, $0.40 \%$ of people with LEL would not have died in the group of people with $0-1$ diseases and $0.89 \%$ in the group of people with $\geq 4$ diseases (Table 2 ).

Figure 2 shows that the background mortality was highest for people with severe MM and the association for education levels off with increasing morbidity.

\section{Association between educational level and premature mortality across MM groups}

We identified 5,170 premature deaths: 2,215 people $(3.2 \%)$ with LEL died prematurely and $36 \%$ of them had $\geq 4$ conditions; 596 people (1.1\%) with HEL died prematurely and $30 \%$ of them had $\geq 4$ conditions. In the group of people with $\geq 4$ conditions, the overall mortality was $14 \%$ higher for people LEL compared with people with HEL (HR: 1.14, 95\% CI: 1.04-1.24), whereas the corresponding estimates for premature mortality as outcome was 40\% (HR: 1.40, 95\% CI: 1.17-1.67) after adjusting for age, sex, cohabitation status, and the 39 conditions (M3). The absolute number of premature deaths was higher among people with LEL than those with HEL in all MM groups: for those with $\geq 4$ diseases, 147 persons with HEL died $\left(\mathrm{CIP}_{1 \mathrm{y}}: 2.99 \%\right)$ versus 803 persons with LEL $\left(\mathrm{CIP}_{1 \mathrm{y}}: 4.17 \%\right)$. Theoretically, if those with LEL had the same risk of dying as those with HEL, $0.24 \%$

Table I Baseline characteristics according to educational level (entire study population)

\begin{tabular}{|c|c|c|c|c|}
\hline \multirow[t]{2}{*}{ Baseline characteristics } & \multirow{2}{*}{$\begin{array}{l}\text { Participants (\%) } \\
(n=239,547)\end{array}$} & \multicolumn{3}{|c|}{ Educational level (\%) } \\
\hline & & $\begin{array}{l}\text { Low }(\leq 10 \text { years }) \\
(n=69,491)\end{array}$ & $\begin{array}{l}\text { Middle }(10-15 \text { years }) \\
(n=\mid 15,597)\end{array}$ & $\begin{array}{l}\text { High }(>15 \text { years }) \\
(n=54,459)\end{array}$ \\
\hline \multicolumn{5}{|l|}{ Age at baseline (years) } \\
\hline $25-34$ & 28,044 (II.7) & $4,317(15.4)$ & $14,732(52.5)$ & $8,995(32.1)$ \\
\hline $35-44$ & $46,222(19.3)$ & $8,009(17.3)$ & 24,904 (53.9) & $13,309(28.8)$ \\
\hline $45-54$ & $50,4 I I(2 I)$ & $12,110(24)$ & $26, \mid 44$ (5I.9) & $12,157(24.1)$ \\
\hline $55-64$ & $5 \mathrm{I}, 277(2 \mathrm{I} .4)$ & $14,456(28.2)$ & $25,485(49.7)$ & $11,336(22.1)$ \\
\hline $65-74$ & $38,162(15.9)$ & I5,886 (4I.6) & $16,324(42.8)$ & $5,952(15.6)$ \\
\hline $75-89$ & $25,431(10.6)$ & $|4,7| 3(57.9)$ & $8,008(31.5)$ & $2,710(10.7)$ \\
\hline \multicolumn{5}{|l|}{ Gender } \\
\hline Female & II,72|7 (48.9) & $30,862(26.3)$ & $63,490(54.2)$ & $22,865(19.5)$ \\
\hline Male & $122,330(51.1)$ & $38,629(31.6)$ & $52,107(42.6)$ & $31,594(25.8)$ \\
\hline \multicolumn{5}{|l|}{ Cohabitation status } \\
\hline Living alone & $72,223(30.1)$ & $27,762(38.4)$ & $30,877(42.8)$ & 13,584 (18.8) \\
\hline Cohabiting & $167,324(69.9)$ & $41,729(24.9)$ & $84,720(50.6)$ & $40,875(24.4)$ \\
\hline \multicolumn{5}{|l|}{ Conditions, number } \\
\hline $0-1$ & $165,643(69.1)$ & $37,743(22.8)$ & $84,326(50.9)$ & $43,574(26.3)$ \\
\hline $2-3$ & $49,085(20.5)$ & $19,199(39.1)$ & $21,813(44.4)$ & $8,073(16.4)$ \\
\hline$\geq 4$ & $24,819(10.4)$ & $12,549(50.6)$ & $9,458(38.1)$ & $2,812(11.3)$ \\
\hline \multicolumn{5}{|l|}{ Psychiatric condition } \\
\hline Yes & $23,519(9.8)$ & $9,293(39.5)$ & $9,887(42)$ & $4,339(18.4)$ \\
\hline
\end{tabular}




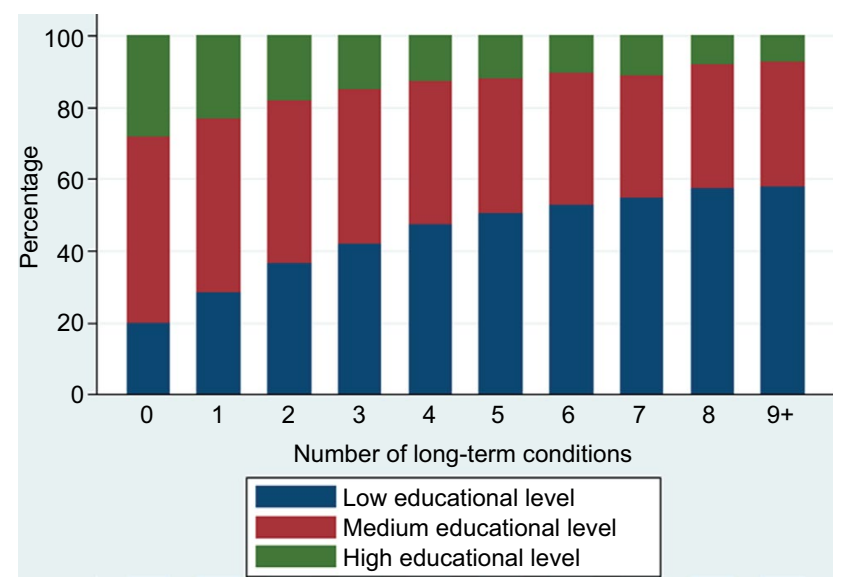

Figure 3 The distribution of educational level relative to the number of long-term conditions. of the people with LEL would not have died in the group of people with $0-1$ conditions and $1.15 \%$ with LEL in the group of people with $\geq 4$ diseases (Table 3 ). Among people with LEL, 2,214 (3.2\%) died prematurely during follow-up and $36 \%$ of them had severe MM. Among people with HEL, $596(1.1 \%)$ died prematurely during follow-up and $25 \%$ of them had severe MM.

\section{Subanalyses}

Effect modification by age and sex

We found that the association between educational level and mortality across MM groups decreased slightly with increasing age (Table S1). The association was similar for men and women (Table S2)

Table 2 Mortality HRs for educational level across multimorbidity groups (entire study population)

\begin{tabular}{|c|c|c|c|c|c|c|c|c|c|c|}
\hline \multirow{2}{*}{$\begin{array}{l}\text { Multimorbidity } \\
\text { group }\end{array}$} & \multirow[t]{2}{*}{ Educational level } & \multirow[t]{2}{*}{ Deaths } & \multirow{2}{*}{$\begin{array}{l}\text { CIP }_{\text {Iy }} \\
(\%)\end{array}$} & \multicolumn{2}{|l|}{$M I^{a}$} & \multicolumn{2}{|l|}{$\mathbf{M}^{\mathrm{b}}$} & \multicolumn{3}{|l|}{$\mathrm{M3}^{\mathrm{c}}$} \\
\hline & & & & HR & $95 \% \mathrm{Cl}$ & HR & $95 \% \mathrm{Cl}$ & HR & $95 \% \mathrm{Cl}$ & Excess CIP $_{\mathrm{Iy}}(\%)^{\mathrm{d}}$ \\
\hline $0-1$ conditions & High $(n=43,574)$ & 335 & 0.14 & 1.00 & Ref & 1.00 & Ref & 1.00 & Ref & Ref \\
\hline \multirow[t]{2}{*}{$(n=165,643)$} & Medium $(n=84,326)$ & 1110 & 0.22 & 1.53 & $(1.36-1.73)$ & 1.45 & $(1.28-1.64)$ & 1.45 & $(1.28-1.64)$ & 0.07 \\
\hline & Low $n=37,743$ ) & 1293 & 0.71 & 2.37 & $(2.10-2.68)$ & 2.27 & $(2.0 \mathrm{I}-2.56)$ & 2.26 & $(2.00-2.55)$ & 0.40 \\
\hline 2-3 conditions & High $(n=8,073)$ & 423 & 1.25 & 1.00 & Ref & 1.00 & Ref & 1.00 & Ref & Ref \\
\hline \multirow[t]{2}{*}{$(n=49,085)$} & Medium $(n=2|, 8| 3)$ & 1486 & 1.63 & 1.25 & $(1.12-1.39)$ & 1.21 & $(1.09-1.35)$ & 1.20 & $(1.08-1.34)$ & 0.27 \\
\hline & Low $(n=19,199)$ & 2069 & 2.62 & 1.38 & $(1.24-1.53)$ & 1.39 & $(1.25-1.54)$ & 1.35 & $(1.22-1.50)$ & 0.68 \\
\hline$\geq 4$ conditions & High $(n=2,8 \mid 2)$ & 514 & 5.59 & 1.00 & Ref & 1.00 & Ref & 1.00 & Ref & Ref \\
\hline \multirow[t]{2}{*}{$(n=2,48 I)$} & Medium $(n=9,458)$ & 2005 & 6.15 & 1.20 & $(1.09-1.32)$ & 1.15 & $(0.05-1.27)$ & 1.12 & $(1.02-1.23)$ & 0.66 \\
\hline & Low $(n=12,549)$ & 3245 & 7.27 & 1.16 & $(1.06-1.28)$ & 1.18 & $(1.08-1.30)$ & 1.14 & $(1.04-1.26)$ & 0.89 \\
\hline
\end{tabular}

Notes: $P$-value $<0.00$ I for no interaction between educational level and multimorbidity group for M3. aAdjusted for age. bike MI with further adjustment for gender and cohabitation status. 'Like M2 with further adjustment for 39 register-based, selected conditions. ${ }^{\mathrm{d}}$ Excess deaths were calculated by multiplying the CIP ${ }_{\text {Iy }}$ by the adjusted attributable fraction (HR-I)/HR for M3.

Abbreviations: $\mathrm{CIP}_{1 y}$, cumulative mortality proportion after I year; $\mathrm{HR}$, hazard ratio; $\mathrm{Cl}$, confidence interval; Ref, reference.

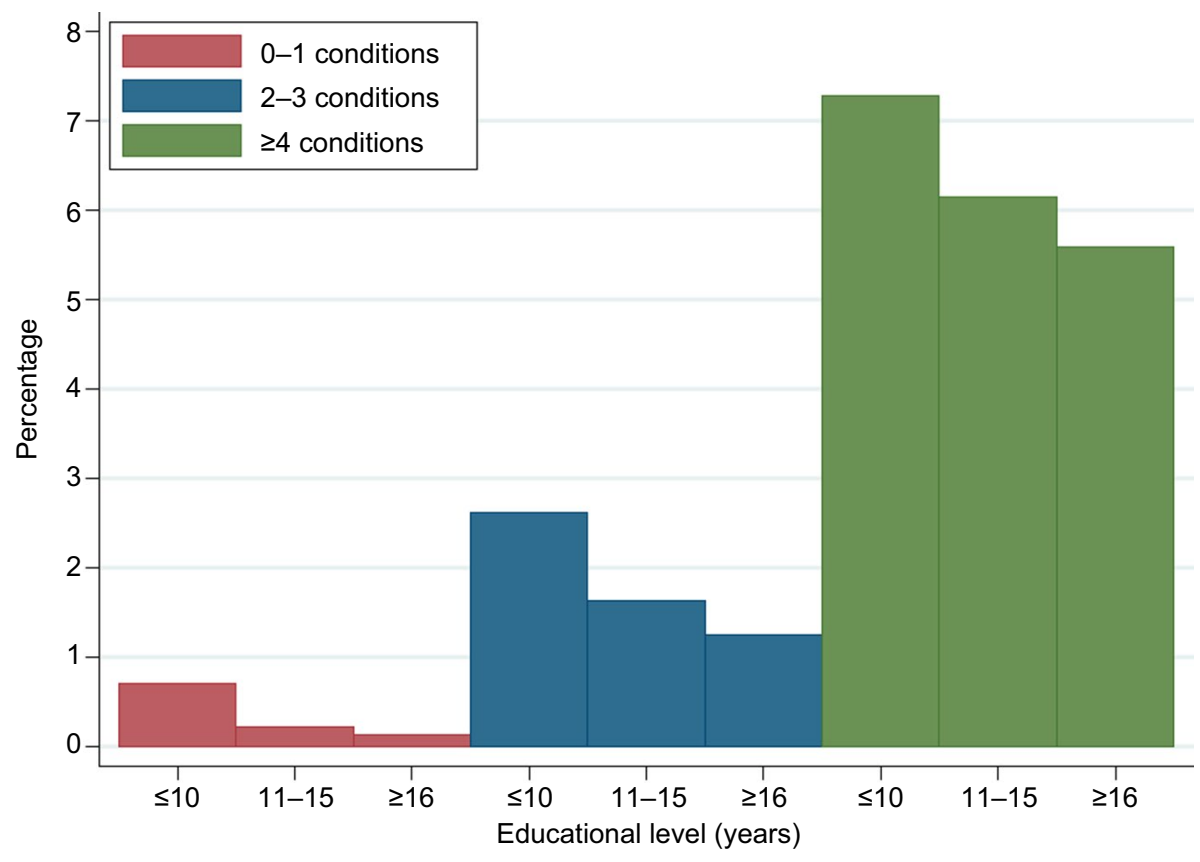

Figure 4 Cumulative incidence proportions of death at I year across educational levels and multimorbidity group. 
Table 3 Premature mortality HRs for educational level across multimorbidity groups (full study population)

\begin{tabular}{|c|c|c|c|c|c|c|c|c|c|c|}
\hline \multirow{2}{*}{$\begin{array}{l}\text { Multimorbidity } \\
\text { group }\end{array}$} & \multirow[t]{2}{*}{ Educational level } & \multirow[t]{2}{*}{ Deaths } & \multirow{2}{*}{$\begin{array}{l}\text { CIP }_{\text {ly }} \\
(\%)\end{array}$} & \multicolumn{2}{|l|}{$M I^{a}$} & \multicolumn{2}{|l|}{$\mathrm{M}^{\mathrm{b}}$} & \multicolumn{3}{|l|}{$M 3^{c}$} \\
\hline & & & & $\overline{H R}$ & $95 \% \mathrm{Cl}$ & $\overline{H R}$ & $95 \% \mathrm{Cl}$ & $\overline{H R}$ & $95 \% \mathrm{Cl}$ & $\overline{\text { Excess } \mathrm{CIP}_{1 y}(\%)^{d}}$ \\
\hline 0-I conditions & High $(n=42,86 I)$ & 249 & 0.12 & 1.00 & Ref & 1.00 & Ref & 1.00 & Ref & Ref \\
\hline \multirow[t]{2}{*}{$(n=159,826)$} & Medium $(\mathrm{n}=82,459)$ & 805 & 0.18 & 1.55 & $(1.35-1.79)$ & 1.45 & $(1.26-1.67)$ & 1.45 & $(1.26-1.67)$ & 0.06 \\
\hline & Low $(n=34,506)$ & 704 & 0.43 & 2.53 & $(2.19-2.93)$ & 2.31 & $(2.00-2.67)$ & 2.31 & $(1.99-2.67)$ & 0.24 \\
\hline 2-3 conditions & High $(n=7,045)$ & 200 & 0.75 & 1.00 & Ref & 1.00 & Ref & 1.00 & Ref & Ref \\
\hline \multirow[t]{2}{*}{$(n=39,603)$} & Medium $(n=18,796)$ & 788 & 1.18 & 1.45 & $(1.24-1.70)$ & 1.40 & $(1.20-1.63)$ & $\mathrm{I} .43$ & $(1.22-1.67)$ & 0.35 \\
\hline & Low $(n=13,762)$ & 708 & 1.50 & 1.67 & $(1.42-1.95)$ & 1.60 & $(1.37-1.88)$ & 1.57 & $(1.34-1.84)$ & 0.54 \\
\hline$\geq 4$ conditions & High $(I, 843)$ & 147 & 2.99 & 1.00 & Ref & 1.00 & Ref & 1.00 & Ref & Ref \\
\hline \multirow[t]{2}{*}{$(n=\mid 4,684)$} & Medium $(6,334)$ & 766 & 4.06 & 1.54 & $(1.29-1.83)$ & 1.45 & $(1.21-1.73)$ & 1.34 & $(1.12-1.60)$ & 1.03 \\
\hline & Low $(6,5 \mid 0)$ & 803 & 4.17 & 1.56 & $(1.31-1.86)$ & 1.52 & $(1.28-1.82)$ & 1.40 & $(1.17-1.67)$ & 1.19 \\
\hline
\end{tabular}

Notes: $P$-value $<0.001$ for no interaction between educational level and multimorbidity group for M3. aAdjusted for age. bike MI with further adjustment for sex and cohabitation status. ' $L i k e \mathrm{M} 2$ with further adjustment for and 39 register-based, selected conditions. ${ }^{\mathrm{d}}$ Excess deaths were calculated by multiplying the $\mathrm{CIP}_{\mathrm{Iy}}$ by the adjusted attributable fraction (HR-I)/HR for M3.

Abbreviations: $\mathrm{CIP}_{1 \mathrm{y}}$, cumulative mortality proportion after I year; $\mathrm{HR}$, hazard ratio; $\mathrm{Cl}$, confidence interval; Ref, reference.

\section{Lifestyle and quality of life in survey respondents}

We performed a subgroup analysis of the survey respondent group with self-reported data on lifestyle and quality of life SF-12 (Figure 1; Table S3). We followed this cohort for a total of 580,646 person-years at risk (mean follow-up time: 3.8 years) and identified 5,875 deaths of which 4,573 (78\%) occurred in people with MM and 2,789 (47\%) occurred in people with LEL.

The association between educational level and mortality disappeared for people with $\mathrm{MM}$ when we adjusted for lifestyle factors and SF-12 (Table S4).

\section{Comparison of respondent and nonrespondent cohorts}

The response rate in the survey was $63 \%$. Nonrespondents were more likely to be male, single, aged 25-45 years or $\geq 75$ years, to have multiple diseases, lower educational level (Table S5), and higher mortality than respondents (Table S6). However, the association between educational level and mortality was remarkably similar for respondents and nonrespondents in most strata of MM (Table S7).

The results were consistent with our main findings when we excluded participants under the age of 35 years (data not shown).

\section{Discussion}

In this population-based cohort study, we confirmed that both LEL and MM were associated with higher overall mortality. ${ }^{19,57}$ Further, our findings suggested that the relative risk of overall and premature mortality for people with LEL compared with HEL decreased with increasing number of underlying chronic conditions.
However, the absolute number of both overall and premature deaths was higher among people with LEL than those with HEL in all MM groups and the highest proportion of deaths that theoretically could have been avoided, given people with LEL had the same mortality as those with HEL, was found in the group of people with $\geq 4$ diseases: both for overall and premature mortality. Among people with LEL, $36 \%$ of those who died prematurely had severe MM, indicating that the association between LEL and higher overall and premature mortality could be driven by MM. In other words, the findings indicate that the relative effect on death decreases the more diseases you suffer from, because the diseases have a direct effect on mortality as seen from the absolute numbers.

Adjusting for lifestyle factors and quality of life (SF-12) eliminated the statistical association between educational level and mortality in people with MM.

\section{Strength and limitations}

We followed a large and representative sample of the Danish population ${ }^{37}$ for up to 4 years with virtually no loss to follow-up. The survey response rate was $63 \%$. Even though the respondent group differed from the nonrespondent group on a range of baseline characteristics, the association between educational level, MM, and mortality was still remarkably similar in the two groups. This finding speaks against selection problems.

The use of national registers with prospectively recorded data ensured highly valid and complete data on deaths, educational level, and baseline socioeconomic factors. ${ }^{58}$ The MM groups were established by an algorithm combining information from Danish national registries with secondary 
care diagnosis and drug prescriptions on an individual level. The algorithm has been described in detail in a previous study of a similar cohort. ${ }^{39} \mathrm{We}$ obtained information on educational level at baseline for $98.8 \%$ of the participants. People with missing data on educational level were equally distributed across gender, age, and MM groups, and we have no reason to believe that the association between educational level and mortality was biased by this approach to missing data. In a subgroup analysis of survey respondents, we further controlled for lifestyle factors and self-rated health. Adverse lifestyle is a well-known prognostic factor for MM and SF-12 can be seen as a proxy for disease severity. The sample size of the study cohort allowed us to investigate mortality at several levels of education and MM and to adjust for many potential confounders. The differences in group sizes reflect the target population, and both our relative and absolute statistical estimates take this into account. Owing to our large study sample, there was sufficient number of events to estimate the effect with each group.

An established definition of SES does not exist in the literature. Krieger et al have described socioeconomic position as referring to the social and economic factors that influence the type of positions that individuals or groups may hold within the structure of a society. ${ }^{59}$ Galobardes et al state that there is no single best indicator of SES that is both suitable for all study aims and yet applicable at all time points in all settings. ${ }^{60}$ In our study, we chose years of schooling as the measure of SES and categorized people into three groups. Education as a proxy for SES is historically founded in Weber's theory of a status domain. ${ }^{61}$ Educational level captures both the transition from parents' SES to own adulthood SES and serves as a determinant for future employment and income. ${ }^{62}$ Additionally, educational level may express a person's ability to navigate in the health care system; it is fixed early in adult life and reported consistently. ${ }^{54}$

Compared to income as a marker of SES, educational level is less volatile, less affected by changes in health status that could impair work capacity, and more stable regarding different use of public benefits such as state educational grants and postemployment benefits.

One important limitation in our study was that the understanding of educational level varies among birth cohorts because considerable changes in educational opportunities have been seen over the recent decades. Hence, a majority of the low educated was found in the older birth cohorts. We addressed this challenge by choosing age as the timescale and letting all individuals enter the study at the same date. Additionally, we performed a stratified analysis after dividing the study population into two age groups of equal size. Another limitation was that we obtained no information about type of education when using years of schooling as SES measure. Furthermore, educational level does not measure human or social capital. The relationship between health and education is complex because formal education is a dynamic process that provides people with multiple resources. Data on educational level from Statistics Denmark were collected prospectively and are regarded as valid. ${ }^{47}$

In the literature, no standard method of measuring MM exists. Consensus is lacking on which conditions to include, how to define the conditions, and whether or not to weight the measure. ${ }^{40}$ The MM status in our study was based on clinically acknowledged conditions from all Danish hospitals and outpatient clinics, including recommended key diseases. ${ }^{63}$ In order to capture common diseases treated in primary care, our algorithm also included redeemed drug prescriptions from all Danish pharmacies. This approach was taken because primary care diagnoses were not available. Several studies on MM use simple disease count models to describe the MM burden. ${ }^{41}$ We weigh the diseases by adjusting for each disease as a dummy variable within each MM stratum; this allows the MM groups to have a different impact on the education level categories due to different combinations of the diseases within each educational level. By doing this, we reduce the residual confounding that may emerge from associating LEL with more severe disease. Yet, we could not account for untreated primary care conditions and the duration or severity of each condition. If people with LEL have an earlier onset of some diseases and more severe states of individual diseases, confounding of the results may occur. We considered people with no chronic diseases and people with one chronic disease as one group. When stratifying on no and one chronic disease, the estimates were not clinically significantly different from each other (data not shown).

In our study, we were able to control for a wide range of potential confounders at the individual level. We were able to adjust for lifestyle factors and quality of life in the survey respondent cohort. We were not able to include information on nutrition that could be a confounder for the association. However, we have adjusted for BMI and other lifestyle factors associated with nutrition. Consequently, the association between educational level and mortality disappeared; this indicates that confounding from these factors may play a role. However, we cannot rule out that some of the factors that we adjusted for are intermediate variables; LEL may lead to an unhealthy lifestyle, which again could lead to higher mortality. If so, adjustment for lifestyle factors could result in 
underestimation of the true association between educational level and mortality. ${ }^{54}$ Even though our study includes many potential confounders, we acknowledge that observational studies may be subject to residual confounding. However, from this finding, it can be discussed how resources should be used. Maybe it would be more reasonable to focus on people having an adverse lifestyle who are at greater risk of developing MM than those who are already multimorbid in order to influence mortality.

\section{Comparison with other studies}

In line with the findings from cross-sectional studies in other countries, we found that MM was associated with LEL. ${ }^{64}$ Our study suggests that the impact of this social determinant on prognosis diminished for people diagnosed with multiple chronic conditions. This finding is consistent with a newly published study from Ontario, Canada, ${ }^{65}$ in which the effect of increasing chronic condition burden on mortality was shown to be similar across neighborhood income quintiles among older people. However, in our study, we were able to look at SES at the individual level across all age groups for 39 long-term conditions. In another study from the UK, the researchers found that the relative socioeconomic inequalities in mortality were smaller at higher levels of morbidity. ${ }^{66}$ They used deprivation categories and included only four chronic diseases. Many studies have investigated the individual impact of educational level and MM on mortality. Yet, as far as we know, our study is the first to contribute with knowledge about the impact of individual educational level on mortality for people with and without MM in a setting with universal health care.

The Danish health care system is predominantly financed through income tax, and universal care is available to all Danish residents. The associations found in our study could possibly be stronger in countries without universal health care or free education.

\section{Conclusion and implications}

Our study suggests that educational level is an important prognostic factor for all-cause mortality and premature mortality. The findings also indicate that this association is modified by level of MM and confounded or modified by lifestyle factors and quality of life. However, the increase in absolute number of deaths for people with LEL compared with people with HEL was greatest in the group of people with severe MM. These findings suggest that both educational level and lifestyle factors must be taken into account when treating people with MM in order to reduce the socioeconomic inequalities in mortality. Increased focus should be directed toward preventing early development of long-term conditions, especially in people with low SES.

\section{Disclosure}

The authors report no conflicts of interest in this work.

\section{References}

1. Lopez AD, Mathers CD. Measuring the global burden of disease and epidemiological transitions: 2002-2030. Ann Trop Med Parasitol. 2006; 100(5-6):481-499.

2. Fortin M, Bravo G, Hudon C, Vanasse A, Lapointe L. Prevalence of multimorbidity among adults seen in family practice. Ann Fam Med. 2005; 3(3):223-228.

3. Uijen AA, van de Lisdonk EH. Multimorbidity in primary care: prevalence and trend over the last 20 years. Eur J Gen Pract. 2008;14(Suppl 1): 28-32.

4. Mercer SW, Gunn J, Bower P, Wyke S, Guthrie B. Managing patients with mental and physical multimorbidity. BMJ. 2012;345:e5559.

5. Cottrell E, Yardley S. Lived experiences of multimorbidity: an interpretative meta-synthesis of patients', general practitioners' and trainees' perceptions.Chronic Illn. 2015;11(4):279-303.

6. Salisbury C. Multimorbidity: redesigning health care for people who use it. Lancet. 2012;380(9836):7-9.

7. Wolff JL, Starfield B, Anderson G. Prevalence, expenditures, and complications of multiple chronic conditions in the elderly. Arch Intern Med. 2002;162(20):2269-2276.

8. Payne RA, Abel GA, Guthrie B, Mercer SW. The effect of physical multimorbidity, mental health conditions and socioeconomic deprivation on unplanned admissions to hospital: a retrospective cohort study. CMAJ. 2013;185(5):E221-E228.

9. Forjaz MJ, Rodriguez-Blazquez C, Ayala A, et al. Chronic conditions, disability, and quality of life in older adults with multimorbidity in Spain. Eur J Intern Med. 2015;26(3):176-181.

10. Alonso-Moran E, Nuno-Solinis R, Orueta JF, Fernandez-Ruanova B, Alday-Jurado A, Gutierrez-Fraile E. Health-related quality of life and multimorbidity in community-dwelling telecare-assisted elders in the Basque country. Eur J Intern Med. 2015;26(3):169-175.

11. Fortin M, Dubois MF, Hudon C, Soubhi H, Almirall J. Multimorbidity and quality of life: a closer look. Health Qual Life Outcomes. 2007; 5:52.

12. Fortin M, Bravo G, Hudon C, et al. Relationship between multimorbidity and health-related quality of life of patients in primary care. Qual Life Res. 2006;15(1):83-91.

13. Lawson KD, Mercer SW, Wyke S, et al. Double trouble: the impact of multimorbidity and deprivation on preference-weighted health related quality of life a cross sectional analysis of the Scottish health survey. Int J Equity Health. 2013;12:67.

14. Barnett K, Mercer SW, Norbury M, Watt G, Wyke S, Guthrie B. Epidemiology of multimorbidity and implications for health care, research, and medical education: a cross-sectional study. Lancet. 2012; 380(9836):37-43.

15. Liddy C, Blazkho V, Mill K. Challenges of self-management when living with multiple chronic conditions: systematic review of the qualitative literature. Can Fam Physician. 2014;60(12):1123-1133.

16. Aarts S, den Akker M, Bosma H, et al. The effect of multimorbidity on health related functioning: Temporary or persistent? Results from a longitudinal cohort study. J Psychosom Res. 2012;73(3):211-217

17. Woo J, Leung J. Multi-morbidity, dependency, and frailty singly or in combination have different impact on health outcomes. Age (Dordr). 2014;36(2):923-931.

18. Eckerblad J, Theander K, Ekdahl A, et al. Symptom burden in community-dwelling older people with multimorbidity: a cross-sectional study. BMC Geriatr. 2015;15:1. 
19. Koroukian SM, Warner DF, Owusu C, Given CW. Multimorbidity redefined: prospective health outcomes and the cumulative effect of co-occurring conditions. Prev Chronic Dis. 2015;12:E55.

20. Caughey GE, Ramsay EN, Vitry AI, et al. Comorbid chronic diseases, discordant impact on mortality in older people: a 14-year longitudinal population study. J Epidemiol Community Health. 2010;64(12):1036-1042.

21. Menotti A, Mulder I, Nissinen A, Giampaoli S, Feskens EJ, Kromhout D. Prevalence of morbidity and multimorbidity in elderly male populations and their impact on 10-year all-cause mortality: the FINE study (finland, italy, netherlands, elderly). J Clin Epidemiol. 2001;54(7):680-686.

22. McLean G, Gunn J, Wyke S, et al. The influence of socioeconomic deprivation on multimorbidity at different ages: a cross-sectional study. Br J Gen Pract. 2014;64(624):e440-e447.

23. Salisbury C, Johnson L, Purdy S, Valderas JM, Montgomery AA. Epidemiology and impact of multimorbidity in primary care: a retrospective cohort study. Br J Gen Pract. 2011;61(582):e12-21.

24. Violan C, Foguet-Boreu Q, Flores-Mateo G, et al. Prevalence, determinants and patterns of multimorbidity in primary care: a systematic review of observational studies. PLoS One. 2014;9(7):e102149.

25. Smith WC, Anderson E, Salinas D, Horvatek R, Baker DP. A metaanalysis of education effects on chronic disease: the causal dynamics of the population education transition curve. SocSci Med. 2015; 127:29-40.

26. Afshar S, Roderick PJ, Kowal P, Dimitrov BD, Hill AG. Multimorbidity and the inequalities of global ageing: a cross-sectional study of 28 countries using the world health surveys. BMC Public Health. 2015; 15:776.

27. van den Akker M, Buntinx F, Metsemakers JF, Roos S, Knottnerus JA. Multimorbidity in general practice: prevalence, incidence, and determinants of co-occurring chronic and recurrent diseases. J Clin Epidemiol. 1998;51(5):367-375.

28. Marengoni A, Winblad B, Karp A, Fratiglioni L. Prevalence of chronic diseases and multimorbidity among the elderly population in Sweden. Am J Public Health. 2008;98(7):1198-1200.

29. Mackenbach JP, Stirbu I, Roskam AJ, et al; European Union Working Group on Socioeconomic Inequalities in Health. Socioeconomic inequalities in health in 22 European countries. $N$ Engl J Med. 2008; 358(23):2468-2481.

30. Madsen M, Andersen AM, Christensen K, Andersen PK, Osler M. Does educational status impact adult mortality in Denmark? A twin approach. Am J Epidemiol. 2010;172(2):225-234.

31. Shkolnikov VM, Andreev EM, Jdanov DA, et al. Increasing absolute mortality disparities by education in Finland, Norway and Sweden, 1971-2000. J Epidemiol Community Health. 2012;66(4):372-378.

32. Mackenbach JP, Bos V, Andersen O, et al. Widening socioeconomic inequalities in mortality in six western European countries. Int J Epidemiol. 2003;32(5):830-837.

33. France EF, Wyke S, Gunn JM, Mair FS, McLean G, Mercer SW. Multimorbidity in primary care: a systematic review of prospective cohort studies. Br J Gen Pract. 2012;62(597):e297-e307.

34. Fortin M, Haggerty J, Almirall J, Bouhali T, Sasseville M, Lemieux M. Lifestyle factors and multimorbidity: a cross sectional study. $B M C$ Public Health. 2014;14:686.

35. Vereecken CA, Maes L, De Bacquer D. The influence of parental occupation and the pupils' educational level on lifestyle behaviors among adolescents in Belgium. J Adolesc Health. 2004;34(4):330-338.

36. van Dam RM, Li T, Spiegelman D, Franco OH, Hu FB. Combined impact of lifestyle factors on mortality: prospective cohort study in US women. BMJ. 2008;337:a1440.

37. Christensen AI, Ekholm O, Glumer C, et al. The danish national health survey 2010. Study design and respondent characteristics. Scand J Public Health. 2012;40(4):391-397.

38. Pedersen CB, Gotzsche H, Moller JO, Mortensen PB. The danish civil registration system. A cohort of eight million persons. Dan Med Bull. 2006;53(4):441-449.
39. Prior A, Fenger-Gron M, Larsen KK, et al. The association between perceived stress and mortality among people with multimorbidity: a prospective population-based cohort study. Am J Epidemiol. 2016;184(3): 199-210.

40. Diederichs C, Berger K, Bartels DB. The measurement of multiple chronic diseases-a systematic review on existing multimorbidity indices. J Gerontol A BiolSci Med Sci. 2011;66(3):301-311.

41. Huntley AL, Johnson R, Purdy S, Valderas JM, Salisbury C. Measures of multimorbidity and morbidity burden for use in primary care and community settings: a systematic review and guide. Ann Fam Med. 2012; 10(2):134-141.

42. Andersen TF, Madsen M, Jorgensen J, Mellemkjoer L, Olsen JH. The danish national hospital register. A valuable source of data for modern health sciences. Dan Med Bull. 1999;46(3):263-268.

43. Gjerstorff ML. The danish cancer registry. Scand J Public Health. 2011; 39(7 Suppl):42-45.

44. Carstensen B, Kristensen JK, Marcussen MM, Borch-Johnsen K. The national diabetes register. Scand J Public Health. 2011;39(7 Suppl): $58-61$.

45. Mors O, Perto GP, Mortensen PB. The danish psychiatric central research register. Scand J Public Health. 2011;39(7 Suppl):54-57.

46. Kildemoes HW, Sorensen HT, Hallas J. The danish national prescription registry. Scand J Public Health. 2011;39(7 Suppl):38-41.

47. Jensen VM, Rasmussen AW. Danish education registers. Scand J Public Health. 2011;39(7 Suppl):91-94.

48. Statistics Denmark. Highest education attained. Available from: http:// www.dst.dk/en/Statistik/dokumentation/documentationofstatistics/ highest-education-attained. Accessed April 24, 2017.

49. UNESCO Institute for Statistics. International Standard Classification of Education; ISCED 2011. Available from: http://www.uis.unesco. org/education/documents/isced-2011-en.pdf. Accessed April 17, 2017.

50. Danish Ministry of Higher Education and Science. The Danish education system. Available at: http://ufm.dk/en/education-and-institutions/ the-danish-education-system. Accessed April 17, 2017.

51. Ware JJr, Kosinski M, Keller SD. A 12-item short-form health survey: construction of scales and preliminary tests of reliability and validity. Med Care. 1996;34(3):220-233.

52. White IR, Royston P, Wood AM. Multiple imputation using chained equations: issues and guidance for practice. Stat Med. 2011;30(4):377-399.

53. Sterne JA, White IR, Carlin JB, et al. Multiple imputation for missing data in epidemiological and clinical research: potential and pitfalls. BMJ. 2009;338:b2393.

54. Rothman K, Greenland S, Lash TL, editors. Modern Epidemiology. 3rd ed. Philadelphia, PA: Lippincott, Williams \& Wilkins; 2008.

55. Andersen PK, Geskus RB, de Witte T, Putter H. Competing risks in epidemiology: possibilities and pitfalls. Int J Epidemiol. 2012;41(3):861-870.

56. Koller MT, Raatz H, Steyerberg EW, Wolbers M. Competing risks and the clinical community: irrelevance or ignorance? Stat Med. 2012; 31(11-12):1089-1097.

57. Osler M, Prescott E. Educational level as a contextual and proximate determinant of all cause mortality in danish adults. J Epidemiol Community Health. 2003;57(4):266-269.

58. Erlangsen A, Fedyszyn I. Danish nationwide registers for public health and health-related research. Scand J Public Health. 2015;43(4):333-339.

59. Krieger N, Williams DR, Moss NE. Measuring social class in US public health research: concepts, methodologies, and guidelines. Annu Rev Public Health. 1997;18:341-378.

60. Galobardes B, Shaw M, Lawlor DA, Lynch JW, Davey Smith G. Indicators of socioeconomic position (part 1). J Epidemiol Community Health. 2006;60(1):7-12.

61. Liberatos P, Link BG, Kelsey JL. The measurement of social class in epidemiology. Epidemiol Rev. 1988;10:87-121.

62. Lynch J KG. Socioeconomic position. In: Berkman LF, Kawachi I, editors. Social Epidemiology. 1st ed. Oxford: Oxford university Press; 2000:13-35. 
63. Huntley AL, Johnson R, Purdy S, Valderas JM, Salisbury C. Measures of multimorbidity and morbidity burden for use in primary care and community settings: a systematic review and guide. Ann Fam Med.2012;10(2):134-141.

64. Violan C, Foguet-Boreu Q, Flores-Mateo G, et al. Prevalence, determinants and patterns of multimorbidity in primary care: a systematic review of observational studies. PLoS One. 2014;9(7):e102149.
65. Lane NE, Maxwell CJ, Gruneir A, Bronskill SE, Wodchis WP. Absence of a socioeconomic gradient in older adults' survival with multiple chronic conditions. EBioMedicine. 2015;2(12):2094-2100.

66. Charlton J, Rudisill C, Bhattarai N, Gulliford M. Impact of deprivation on occurrence, outcomes and health care costs of people with multiple morbidity. J Health Serv Res Policy. 2013;18(4):215-223.
Clinical Epidemiology

\section{Publish your work in this journal}

Clinical Epidemiology is an international, peer-reviewed, open access, online journal focusing on disease and drug epidemiology, identification of risk factors and screening procedures to develop optimal preventative initiatives and programs. Specific topics include: diagnosis, prognosis, treatment, screening, prevention, risk factor modification,

Submit your manuscript here: https://www.dovepress.com/clinical-epidemiology-journal

\section{Dovepress}

systematic reviews, risk and safety of medical interventions, epidemiology and biostatistical methods, and evaluation of guidelines, translational medicine, health policies and economic evaluations. The manuscript management system is completely online and includes a very quick and fair peer-review system, which is all easy to use. 